\title{
The Structure Theorem for The cut locus of a Certain Class of Cylinders of Revolution I *!
}

\author{
Pakkinee CHITSAKUL
}

\begin{abstract}
The aim of this paper is to determine the structure of the cut locus for a class of surfaces of revolution homeomorphic to a cylinder. Let $M$ denote a cylinder of revolution which admits a reflective symmetry fixing a parallel called the equator of $M$. It will be proved that the cut locus of a point $p$ of $M$ is a subset of the union of the meridian and the parallel opposite to $p$ respectively, if the Gaussian curvature of $M$ is decreasing on each upper half meridian.
\end{abstract}

\section{Introduction}

It is a very difficult problem to determine the structure of the cut locus of a Riemannian manifold and it was difficult even for a quadric surface.

Since Elerath $([\mathrm{E}])$ succeeded in specifying the structure of the cut locus for paraboloids of revolution and (2-sheeted) hyperboloids of revolution, the structures of the cut locus for quadric surfaces of revolution have been studied. After his work, Sinclair and Tanaka ([ST]) determined the structure of the cut locus for a class of surfaces of revolution containing the ellipsoids. Notice that the structures of the cut locus for triaxial ellipsoids with unequal axes were also determined by Itoh and Kiyohara ([IK]).

On the structure of the cut locus for a cylinder of revolution $\left(R^{1} \times S^{1}, d t^{2}+m(t)^{2} d \theta^{2}\right)$, Tsuji ([Ts] ) first determined the cut locus of a point on the equator $t=0$ if the cylinder is symmetric with respect to the equator and the Gaussian curvature is decreasing on the upper half meridian $t>0, \theta=0$. In 2003, Tamura ([Ta]) determined the structure of the cut locus by adding an assumption $m^{\prime} \neq 0$ except $t=0$. In this paper, we determine the structure of the cut locus without this assumption.

Here, let us review the notion of a cut point and the cut locus of a point. Let $\gamma$ : $[0, a] \rightarrow M$ be a minimal geodesic segment in a complete Riemannian manifold $M$. The end point of $\gamma(a)$ is called a cut point of $\gamma(0)$ along $\gamma$, if any geodesic extension of $\gamma$ is not minimal anymore. The cut locus $C_{p}$ of a point $p$ of $M$ is by definition the set of the cut points along all minimal geodesic segments emanating from $p$.

In this paper we will prove the following theorem.

*Mathematics Subject Classification (2010): 53C22.

${ }^{\dagger}$ Keywords: cut point, cut locus, cylinder of revolution 
Main Theorem Let $\left(M, d s^{2}\right)$ be a complete Riemannian manifold $R^{1} \times S^{1}$ with a warped product metric $d s^{2}=d t^{2}+m(t)^{2} d \theta^{2}$ of the real line $\left(R^{1}, d t^{2}\right)$ and the unit circle $\left(S^{1}, d \theta^{2}\right)$. Suppose that the warping function $m$ is a positive-valued even function and the Gaussian curvature of $M$ is decreasing along the half meridian $t^{-1}[0, \infty) \cap \theta^{-1}(0)$. If the Gaussian curvature of $M$ is positive on $t=0$, then the structure of the cut locus $C_{q}$ of a point $q \in \theta^{-1}(0)$ in $M$ is given as follows:

1. The cut locus $C_{q}$ is the union of a subarc of the parallel $t=-t(q)$ opposite to $q$ and the meridian opposite to $q$ if $|t(q)|<t_{0}:=\sup \left\{t>0 \mid m^{\prime}(t)<0\right\}$ and $\varphi(m(t(q)))<\pi$. More precisely,

$$
C_{q}=\theta^{-1}(\pi) \cup\left(t^{-1}(-t(q)) \cap \theta^{-1}[\varphi(m(t(q))), 2 \pi-\varphi(m(t(q)))]\right) .
$$

2. The cut locus $C_{q}$ is the meridian $\theta^{-1}(\pi)$ opposite to $q$ if $\varphi(m(t(q))) \geq \pi$ or if $|t(q)| \geq t_{0}$.

Here, the function $\varphi(\nu)$ on $(\inf m, m(0))$ is defined as

$$
\varphi(\nu):=2 \int_{-\xi(\nu)}^{0} \frac{\nu}{m \sqrt{m^{2}-\nu^{2}}} d t=2 \int_{0}^{\xi(\nu)} \frac{\nu}{m \sqrt{m^{2}-\nu^{2}}} d t,
$$

where $\xi(\nu):=\min \{t>0 \mid m(t)=\nu\}$. Notice that the point $q$ is an arbitrarily given point if the coordinates $(t, \theta)$ are chosen so as to satisfy $\theta(q)=0$.

Remark 1.1 If the Gaussian curvature of a cylinder of revolution is nonpositive everywhere, then any geodesic has no conjugate point. Therefore, it is clear to see that the cut locus of a point on the manifold is the meridian opposite to the point.

\section{Preliminaries}

Let $f$ be the solution of the differential equation

$$
f^{\prime \prime}+K f=0
$$

with initial conditions $f(0)=c$ and $f^{\prime}(0)=0$. Here $c$ denotes a fixed positive number and $K:[0, \infty) \rightarrow R$ denotes a continuous function.

Lemma 2.1 If $K(0)>0$ and $f^{\prime}(t) \neq 0$ for any $t>0$, then $f^{\prime}(t)<0$ on $(0, \infty)$. Furthermore, if $f>0$ on $[0, \infty)$, then $K(t)<0$ for some $t>0$.

Proof. Since $f^{\prime \prime}(0)=-K(0) f(0)<0$ by (2.1), $f^{\prime}(t)$ is strictly decreasing on $(0, \delta)$ for some $\delta>0$. This implies that $0=f^{\prime}(0)>f^{\prime}(t)$ for any $t \in(0, \delta)$. Since $f^{\prime} \neq 0$ on $[0, \infty)$, $f^{\prime}(t)<0$ on $(0, \infty)$. Furthermore, we assume that $f>0$ on $[0, \infty)$. Supposing that $K \geq 0$ on $[0, \infty)$, we will get a contradiction. By (2.1),

$$
f^{\prime \prime}(t)=-K(t) f(t) \leq 0
$$

on $[0, \infty)$. Hence $f^{\prime}(t)$ is decreasing on $[0, \infty)$. In particular, $0=f^{\prime}(0)>f^{\prime}(\delta) \geq f^{\prime}(t)$ for any $t \geq \delta$. This contradicts the assumption $f>0$. 
Lemma 2.2 Suppose that $K(0)>0$ and $f>0$ on $[0, \infty)$. If $f^{\prime}(t)=0$ for some $t>0$ and $K$ is decreasing, then there exist a unique solution $t=t_{0} \in(0, \infty)$ of $f^{\prime}(t)=0$ such that $f^{\prime}(t)<0$ on $\left(0, t_{0}\right)$ and $f^{\prime}(t)>0$ on $\left(t_{0}, \infty\right)$ and there exists $t_{1} \in\left(0, t_{0}\right)$ satisfying $K\left(t_{1}\right)=0$. Hence $K \geq 0$ on $\left[0, t_{1}\right]$ and $K \leq 0$ on $\left[t_{1}, \infty\right)$.

Proof. Let $a>0$ denote the minimum positive solution $t=a$ of $f^{\prime}(t)=0$. Suppose that there exist another solution $b(>a)$ satisfying $f^{\prime}(b)=0$. By the mean value theorem, there exist $t_{1} \in(0, a)$ and $s_{1} \in(a, b)$ satisfying $f^{\prime \prime}\left(t_{1}\right)=f^{\prime \prime}\left(s_{1}\right)=0$. Hence $K\left(t_{1}\right)=K\left(s_{1}\right)=0$ by (2.1). Since $K$ is decreasing, $K=0$ on $\left[t_{1}, s_{1}\right]$. Therefore, by (2.1), $f^{\prime \prime}(t)=0$ on $\left[t_{1}, s_{1}\right]$. In particular, $f^{\prime}(a)=f^{\prime}\left(t_{1}\right)=0$. Since $0<t_{1}<a, t_{1}$ is a positive solution $t$ of $f^{\prime}(t)=0$, which is less than $a$. This is a contradiction. Therefore, there exists a unique positive solution $t=t_{0}$ of $f^{\prime}(t)=0$. From the mean value theorem and (2.1), there exists $t_{1} \in\left(0, t_{0}\right)$ satisfying $K\left(t_{1}\right)=0$. Since $K(t)$ is decreasing, $K \geq 0$ on $\left[0, t_{1}\right]$ and $K \leq 0$ on $\left[t_{1}, \infty\right)$. Hence by (2.1), $f^{\prime \prime}(t)=-K(t) f(t) \geq 0$ on $\left[t_{1}, \infty\right)$ and $f^{\prime}(t) \geq f^{\prime}\left(t_{0}\right)=0$ for any $t>t_{0}$. Since $f^{\prime}$ has a unique positive zero, $f^{\prime}>0$ on $\left(t_{0}, \infty\right)$. It is clear from the proof of Lemma 2.1 that $f^{\prime}<0$ on $\left(0, t_{0}\right)$.

\section{Review of the behavior of geodesics}

From now on, $M$ denotes a complete Riemannian manifold $R^{1} \times S^{1}$ with a warped product Riemannian metric $d s^{2}=d t^{2}+m(t)^{2} d \theta^{2}$ of the real line $\left(R^{1}, d t^{2}\right)$ and the unit circle $\left(S^{1}, d \theta^{2}\right)$. Let us review the behavior of a geodesic $\gamma(s)=(t(s), \theta(s))$ on the manifold $M$. For each unit speed geodesic $\gamma(s)=(t(s), \theta(s))$, there exists a constant $\nu$ satisfying

$$
m(t(s))^{2} \theta^{\prime}(s)=\nu .
$$

Hence, if $\eta(s)$ denotes the angle made by the velocity vector $\gamma^{\prime}(s)$ of the geodesic $\gamma(s)$ and the tangent vector $(\partial / \partial \theta)_{\gamma(s)}$, then

$$
m(t(s)) \cos \eta(s)=\nu
$$

for any s. The constant $\nu$ is called the Clairaut constant of $\gamma$. The reader should refer to Chapter 7 in [SST] for the Clairaut relation. Since $\gamma(s)$ is unit speed,

$$
t^{\prime}(s)^{2}+m(t(s))^{2} \theta^{\prime}(s)^{2}=1
$$

holds. By (3.1) and (3.3), it follows that

$$
\begin{gathered}
t^{\prime}(s)= \pm \frac{\sqrt{m(t(s))^{2}-\nu^{2}}}{m(t(s))} \\
\theta\left(s_{2}\right)-\theta\left(s_{1}\right)=\varepsilon\left(t^{\prime}(s)\right) \int_{t\left(s_{1}\right)}^{t\left(s_{2}\right)} \frac{\nu}{m \sqrt{m^{2}-\nu^{2}}} d t
\end{gathered}
$$

holds, if $t^{\prime}(s) \neq 0$ on $\left(s_{1}, s_{2}\right)$ and $\varepsilon\left(t^{\prime}(s)\right)$ denotes the sign of $t^{\prime}(s)$. 
The length $L(\gamma)$ of a geodesic segment $\gamma(s)=(t(s), \theta(s)), s_{1} \leq s \leq s_{2}$ is

$$
L(\gamma)=\varepsilon\left(t^{\prime}(s)\right) \int_{t\left(s_{1}\right)}^{t\left(s_{2}\right)} \frac{m(t)}{\sqrt{m()^{2}-\nu^{2}}} d t
$$

if $t^{\prime}(s) \neq 0$ on $\left(s_{1}, s_{2}\right)$.

From a direct computation, the Gaussian curvature $G$ of $M$ is given by

$$
G(q)=-\frac{m^{\prime \prime}}{m}(t(q))
$$

at each point $q \in M$. Since $G$ is constant on $t^{-1}(a)$ for each $a \in R$, a smooth function $K$ on $R$ is defined by

$$
K(u):=G(q)
$$

for $q \in t^{-1}(u)$. Therefore $m$ satisfies the following differential equation

$$
m^{\prime \prime}+K m=0
$$

with $m^{\prime}(0)=0$.

From now on, we assume that the Gaussian curvature $G$ of $M$ is positive on $t^{-1}(0)$, and $m(t)=m(-t)$ holds for any $t \in R$. Hence, $M$ is symmetric with respect to the equator $t=0$ and if $K$ is decreasing on [0,, , then by Lemma 2.2. $m^{\prime}(t)<0$ for all $t>0$ or there exists a unique positive solution $t=t_{0}$ of $m^{\prime}(t)=0$ such that $m^{\prime}<0$ on $\left(0, t_{0}\right)$ and $m^{\prime}>0$ on $\left(t_{0}, \infty\right)$. Furthermore, if the latter case happens, there exists $t_{1} \in\left(0, t_{0}\right)$ such that $K \geq 0$ on $\left[0, t_{1}\right]$ and $K \leq 0$ on $\left[t_{1}, \infty\right)$.

For technical reasons, we treat both geodesics on $M$ and its universal covering space $\pi: \widetilde{M} \rightarrow M$, where $\widetilde{M}:=\left(R^{1} \times R^{1}, d \tilde{t}^{2}+m(\tilde{t})^{2} d \tilde{\theta}^{2}\right)$.

Choose any point $p$ on the equator $t=0$. We may assume that $\theta(p)=0$ without loss of generality. Let $\gamma:[0, \infty) \rightarrow M$ denote a geodesic emanating from $p=\gamma(0)$ with Clairaut constant $\nu \in(\inf m, m(0))$. Notice that $\gamma$ is uniquely determined up to the reflection with respect to $t=0$. The geodesic $\gamma(s)=(t(s), \theta(s))$ is tangent to the parallel $t=\xi(\nu)$ ( if $\left.t \circ \gamma)^{\prime}(0)>0\right)$ or $t=-\xi(\nu)\left(\right.$ if $\left.(t \circ \gamma)^{\prime}(0)<0\right)$, where $\xi(\nu)>0$ denotes the least positive solution of $m(\xi(\nu))=\nu$, that is,

$$
\xi(\nu):=\min \{u>0 \mid m(u)=\nu\} .
$$

After $\gamma$ is tangent to the parallel $t=\xi(\nu)$ or $-\xi(\nu), \gamma$ intersects the equator $t=0$ again. Thus, after $\tilde{\gamma}$ is tangent to the parallel arc $\tilde{t}=\xi(\nu)$ or $-\xi(\nu), \tilde{\gamma}$ intersect $\tilde{t}=0$ again. Here $\tilde{\gamma}$ denotes a geodesic on $\widetilde{M}$ satisfying $\gamma=\pi \circ \tilde{\gamma}$.

From (3.5), we obtain,

$$
\tilde{\theta}\left(s_{0}\right)-\tilde{\theta}(0)=\int_{-\xi(\nu)}^{0} \frac{\nu}{m \sqrt{m^{2}-\nu^{2}}} d t=\int_{0}^{\xi(\nu)} \frac{\nu}{m \sqrt{m^{2}-\nu^{2}}} d t,
$$

and

$$
\tilde{\theta}\left(s_{1}\right)-\tilde{\theta}\left(s_{0}\right)=\int_{-\xi(\nu)}^{0} \frac{\nu}{m \sqrt{m^{2}-\nu^{2}}} d t=\int_{0}^{\xi(\nu)} \frac{\nu}{m \sqrt{m^{2}-\nu^{2}}} d t,
$$

where $s_{0}:=\min \{s>0 \mid m(\tilde{t}(s))=\nu\}, s_{1}:=\min \{s>0 \mid \tilde{t}(s)=0\}$.

By summing up the argument above, we have, 
Lemma 3.1 Let $\tilde{\gamma}(s)=(\tilde{t}(s), \tilde{\theta}(s))$ denote a geodesic emanating from the point $\tilde{p}:=$ $(\tilde{t}, \tilde{\theta})^{-1}(0,0)$ with Clairaut constant $\nu \in(\inf m, m(0))$. Then $\tilde{\gamma}$ intersects $\tilde{t}=0$ again at the point $(\tilde{t}, \tilde{\theta})^{-1}(0, \varphi(\nu))$. Here,

$$
\varphi(\nu):=2 \int_{-\xi(\nu)}^{0} \frac{\nu}{m \sqrt{m^{2}-\nu^{2}}} d t=2 \int_{0}^{\xi(\nu)} \frac{\nu}{m \sqrt{m^{2}-\nu^{2}}} d t .
$$

Lemma 3.2 The length $l(\nu)$ of the $\operatorname{subarc}(\tilde{t}(s), \tilde{\theta}(s)), 0 \leq \tilde{\theta}(s) \leq \varphi(\nu)$, of $\tilde{\gamma}(s)$ is given by

$$
l(\nu)=2 \int_{-\xi(\nu)}^{0} \frac{m}{\sqrt{m^{2}-\nu^{2}}} d t=2 \int_{-\xi(\nu)}^{0} \frac{\sqrt{m^{2}-\nu^{2}}}{m} d t+\nu \varphi(\nu)
$$

and

$$
\frac{\partial l}{\partial \nu}(\nu)=\nu \varphi^{\prime}(\nu)
$$

Proof. From (3.6), we obtain,

$$
l(\nu)=2 \int_{-\xi(\nu)}^{0} \frac{m}{\sqrt{m^{2}-\nu^{2}}} d t .
$$

Since

$$
\frac{m}{\sqrt{m^{2}-\nu^{2}}}=\frac{\sqrt{m^{2}-\nu^{2}}}{m}+\frac{\nu^{2}}{m \sqrt{m^{2}-\nu^{2}}}
$$

holds, we get

$$
l(\nu)=2 \int_{-\xi(\nu)}^{0} \frac{\sqrt{m^{2}-\nu^{2}}}{m} d t+2 \int_{-\xi(\nu)}^{0} \frac{\nu^{2}}{m \sqrt{m^{2}-\nu^{2}}} d t .
$$

Hence, by (3.7), we get (3.8). By differentiating $l(\nu)$ with respect to $\nu$, we get,

$$
l^{\prime}(\nu)=2 \int_{-\xi(\nu)}^{0} \frac{\partial}{\partial \nu} \frac{\sqrt{m^{2}-\nu^{2}}}{m} d t+\varphi(\nu)+\nu \varphi^{\prime}(\nu)=\nu \varphi^{\prime}(\nu) .
$$

\section{The decline of the function $\varphi(\nu)$}

Let $\pi: \widetilde{M}=\left(R^{1} \times R^{1}, d \tilde{t}^{2}+m(\tilde{t})^{2} d \tilde{\theta}^{2}\right) \rightarrow M$ denote the universal covering space of $M$. We choose an arbitrary point $\tilde{p}$ of $\tilde{t}^{-1}(-\infty, 0]$, and we denote the cut locus of $\tilde{p}$ by $C_{\tilde{p}}$. Before proving some lemmas on the cut locus, let us review the structure of the cut locus of $\widetilde{M}$. We refer to $[\mathrm{ShT}]$ or $[\mathrm{SST}]$ on the structure of the cut locus of a 2-dimensional complete Riemannian manifold.

It is known that the cut locus has a local tree structure. Since $\widetilde{M}$ is simply connected, the cut locus has no circle. If two cut points $x$ and $y$ are in a common connected component of the cut locus, then $x$ and $y$ are connected by a unique rectifiable arc in the cut locus. 
Since $\widetilde{M}$ is homeomorphic to $R^{2}$, we may define a global sector at each cut point. For general surfaces, only local sectors are defined (see [ShT], or [SST]). A global sector at each cut point $x$ of the point $\tilde{p}$ is by definition a connected component of $\widetilde{M} \backslash \Gamma_{x}$, where $\Gamma_{x}$ denotes the set of all points lying on a minimal geodesic segment joining $\tilde{p}$ to $x$. Let $c:[0, a] \rightarrow C_{\tilde{p}}$ denote a rectifiable arc in the cut locus. Then for each cut point $c(t), t \in(0, a), c$ bisects the sector at $c(t)$ containing $c[0, t)$ (respectively $c(t, a])$. For each sector of the point $\tilde{p}$ on $\widetilde{M}$, there exists an end point of $C_{\tilde{p}}$, since $C_{\tilde{p}}$ has no circle. Here, a cut point $q$ of $\tilde{p}$ is called an end point if $q$ admits exactly one sector.

In this section, we assume that the Gaussian curvature $G$ of $M$ is increasing on the half meridian $t^{-1}(-\infty, 0] \cap \theta^{-1}(0)$ and that $M$ has a reflective symmetry with respect to $t=0$. Hence the Gaussian curvature of $\widetilde{M}$ is increasing on the lower half meridian $\tilde{t}^{-1}(-\infty, 0] \cap \tilde{\theta}^{-1}(0)$ and $\widetilde{M}$ has a reflective symmetry with respect to $\tilde{t}=0$.

Lemma 4.1 Suppose that there exists a cut point of the point $\tilde{p}$ in $\tilde{t}^{-1}(-\infty, 0)$. Then there exist two minimal geodesic segments $\alpha$ and $\beta$ joining $\tilde{p}$ to a cut point $y$ of $\tilde{p}$ such that the global sector $D(\alpha, \beta)$ bounded by $\alpha$ and $\beta$ has an end point of $C_{\tilde{p}}$ and $D(\alpha, \beta) \subset$ $\tilde{t}^{-1}(-\infty, 0)$.

Proof. Since the subset of cut points admitting at least two minimal geodesics is dense in the cut locus, the existence of two minimal geodesics $\alpha$ and $\beta$ is clear (see $[\mathrm{Bh}]$ ). Since $\widetilde{M}$ has a reflective symmetry with respective to $\tilde{t}=0$, it is trivial that $D(\alpha, \beta) \subset \tilde{t}^{-1}(-\infty, 0)$. Let $y$ denote the end point of $\alpha$ distinct from $\tilde{p}$. Since the proof is complete in the case where the cut point $y$ is not an end point of the cut locus, we assume that $y$ is an end point. Then, we get an $\operatorname{arc} c$ in the cut locus emanating from $y$. Any interior point $y_{1}$ on $c$ is not an end point of the cut locus. It is clear that there exist two minimal geodesic segments joining $\tilde{p}$ and $y_{1}$ which bound a sector containing $y$ as an end point of the cut locus.

Lemma 4.2 For any unit speed minimal geodesic segment $\gamma:[0, L(\gamma)] \rightarrow \widetilde{M}$ joining $\tilde{p}$ to any end point $x$ of $C_{\tilde{p}}$ in the domain $D(\alpha, \beta), x$ is conjugate to $\tilde{p}$ along $\gamma$ and $\gamma$ is shorter than $\alpha$ and $\beta$.

Proof. Note that for any end point $x$ of the cut locus, the set of all minimal geodesic segments joining $\tilde{p}$ to $x$ is connected. Therefore, $x$ is conjugate to $\tilde{p}$ along any minimal geodesic segments joining $\tilde{p}$ to the end point of the cut locus. Let $\gamma:[0, L(\gamma)] \rightarrow \widetilde{M}$ denote any minimal geodesic segment $\tilde{p}$ to an end point $x$ of $C_{\tilde{p}} \cap D(\alpha, \beta)$. We will prove that $\gamma$ is shorter than $\alpha$ and $\beta$. It follows from Theorem B in [ShT] or [IT] that there exists a unit speed $\operatorname{arc} c:[0, l] \rightarrow C_{\tilde{p}}$ joining the end point $x$ to $y$, where $y$ denotes the end point of $\alpha$ distinct from $\tilde{p}$. Since the function $d(\tilde{p}, c(\tau))$ is a Lipschitz function, it follows from Lemma 7.29 in [WZ that the function is differentiable for almost all $\tau$ and

$$
d(\tilde{p}, c(l))-d(\tilde{p}, y)=\int_{0}^{l} \frac{d}{d \tau} d(\tilde{p}, c(\tau)) d \tau
$$

holds. From the Clairaut relation (3.2), the inner angle $\theta(\tau)$ at $c(\tau)$ of the sector containing 
$c[0, \tau)$ is less than $\pi$. Hence, by the first variation formula, we get

$$
\frac{d}{d \tau} d(\tilde{p}, c(\tau))=\cos \frac{\theta(\tau)}{2}>0
$$

for almost all $\tau$. Notice that for each each $\tau \in(0, l)$, the curve $c$ bisects the sector at $c(\tau)$ containing $c[0, \tau)$. Therefore, from (4.1),

$$
L(\alpha)=L(\beta)=d(\tilde{p}, c(l))>d(\tilde{p}, y)=L(\gamma) .
$$

Lemma 4.3 Let $q$ be a point on $\tilde{\theta}^{-1}(0)$ and $u_{0}$ any real number. Then $d(q, c(\theta))$ is strictly increasing on $[0, \infty)$. Here $c:[0, \infty) \rightarrow \widetilde{M}$ denotes $c(\theta)=\left(u_{0}, \theta\right)$ in the coordinates $(\tilde{t}, \tilde{\theta})$ and $d(\cdot, \cdot)$ denotes the Riemannian distance function on $\widetilde{M}$.

Proof. Choose any positive numbers $\theta_{1}<\theta_{2}$. Let $\alpha_{i}, i=1,2$, denote minimal geodesic segments joining the point $q$ to $c\left(\theta_{i}\right)$ respectively. Since $\theta_{2}>\theta_{1}$, there exists an intersection $\alpha_{2}\left(t_{2}\right)$ of $\alpha_{2}$ and the meridian $\tilde{\theta}=\theta_{1}$. The point $c\left(\theta_{1}\right)$ is the unique nearest point on $\tilde{t}=u_{0}$ from $\alpha_{2}\left(t_{2}\right)$. Hence,

$$
d\left(\alpha_{2}\left(t_{2}\right), c\left(\theta_{1}\right)\right)<d\left(\alpha_{2}\left(t_{2}\right), c\left(\theta_{2}\right)\right) .
$$

Therefore, by the triangle inequality, we get

$$
d\left(q, c\left(\theta_{2}\right)\right)=d\left(q, \alpha_{2}\left(t_{2}\right)\right)+d\left(\alpha_{2}\left(t_{2}\right), c\left(\theta_{2}\right)\right)>d\left(q, \alpha_{2}\left(t_{2}\right)\right)+d\left(\alpha_{2}\left(t_{2}\right), c\left(\theta_{1}\right)\right) \geq d\left(q, c\left(\theta_{1}\right)\right) .
$$

This implies that $d(q, c(\theta))$ is strictly increasing on $[0, \infty)$.

Lemma 4.4 Suppose that $\gamma:[0, L(\gamma)] \rightarrow \widetilde{M}$ is a minimal geodesic segment joining $\tilde{p}$ to an end point $x \in C_{\tilde{p}}$, which is a point in the sector $D(\alpha, \beta)$ bounded by two minimal geodesic segments $\alpha$ and $\beta$ emanating from $\tilde{p}$. Then, for any $s \in[0, L(\gamma)], \tilde{t}(\alpha(s)) \geq$ $\tilde{t}(\gamma(s)) \geq \tilde{t}(\beta(s))$ holds. Here we assume that

$$
\angle\left(\alpha^{\prime}(0),(\partial / \partial \tilde{t})_{\tilde{p}}\right)<\angle\left(\gamma^{\prime}(0),(\partial / \partial \tilde{t})_{\tilde{p}}\right)<\angle\left(\beta^{\prime}(0),(\partial / \partial \tilde{t})_{\tilde{p}}\right)
$$

where $\angle(\cdot, \cdot)$ denotes the angle made by two tangent vectors.

Proof. From (3.4), it follows that for sufficiently small $s>0, \tilde{t}(\alpha(s))>\tilde{t}(\gamma(s))>\tilde{t}(\beta(s))$ holds. Hence the set $A:=\{s \in(0, L(\gamma)) \mid \tilde{t}(\alpha(s))>\tilde{t}(\gamma(s))>\tilde{t}(\beta(s))\}$ is a nonempty open subset of $(0, L(\gamma))$. Let $\left(0, s_{0}\right)$ denote the connected component of $A$. It is sufficient to prove that $s_{0}=L(\gamma)$. Suppose that $s_{0}<L(\gamma)$. Thus, $\tilde{t}\left(\alpha\left(s_{0}\right)\right)=\tilde{t}\left(\gamma\left(s_{0}\right)\right)$ or $\tilde{t}\left(\gamma\left(s_{0}\right)\right)=\tilde{t}\left(\beta\left(s_{0}\right)\right)$ holds, since $A$ is open. By applying Lemma 4.3 for $u_{0}:=\tilde{t}\left(\alpha\left(s_{0}\right)\right)$ and $\tilde{t}\left(\beta\left(s_{0}\right)\right)$, we get $\alpha\left(s_{0}\right)=\gamma\left(s_{0}\right)$ or $\gamma\left(s_{0}\right)=\beta\left(s_{0}\right)$, which is a contradiction.

Lemma 4.5 For any point $\tilde{p} \in \tilde{t}^{-1}(-\infty, 0]$, there does not exist a cut point of $\tilde{p}$ in $\tilde{t}^{-1}(-\infty, 0)$. In particular, the cut locus of $\tilde{p}$ is a subset of $\tilde{t}^{-1}(0)$ if $\tilde{t}(\tilde{p})=0$. This implies that the cut locus $C_{p}$ of a point $p \in t^{-1}(0)$ is a subset of $\theta^{-1}(\pi) \cup t^{-1}(0)$. Here the coordinates $(t, \theta)$ are chosen so as to satisfy $\theta(p)=0$. 
Proof. Suppose that there exist a cut point of $\tilde{p}$ in $\tilde{t}^{-1}(-\infty, 0)$. By Lemma 4.1, there exist two minimal geodesic segments $\alpha$ and $\beta$ joining a cut point $y$ of $\tilde{p}$ which bound a sector $D(\alpha, \beta)$ containing an end point $x$ of $C_{\tilde{p}}$. Let $\gamma:[0, L(\gamma)] \rightarrow \widetilde{M}$ be a unit speed geodesic segment joining $\tilde{p}$ to the end point $x$. From Lemmas 4.1 and 4.4 , it follows that for any $s \in[0, L(\gamma)]$,

$$
0 \geq \tilde{t}(\alpha(s)) \geq \tilde{t}(\gamma(s)) \geq \tilde{t}(\beta(s))
$$

holds. Since the Gaussian curvature $G$ is increasing on each lower half meridian, we obtain

$$
G(\alpha(s)) \geq G(\gamma(s)) \geq G(\beta(s)) .
$$

By applying the Rauch comparison theorem for the pair of geodesic segments $\left.\alpha\right|_{[0, L(\gamma)]}$ and $\gamma, \tilde{p}$ admits a conjugate point on $\left.\alpha\right|_{[0, L(\gamma)]}$ along $\alpha$.

This contradicts the fact that $\alpha$ is minimal. Since $\widetilde{M}$ is symmetric with respect to $\tilde{t}=$ 0 , the cut locus of $\tilde{p}$ is a subset of $\tilde{t}^{-1}(0)$, if $\tilde{t}(\tilde{p})=0$. This implies that $C_{p} \subset \theta^{-1}(\pi) \cup t^{-1}(0)$ for the point $p=t^{-1}(0) \cap \theta^{-1}(0)$.

Proposition 4.6 Let $M$ be a complete Riemannian manifold $R^{1} \times S^{1}$ with a warped product metric $d s^{2}=d t^{2}+m(t)^{2} d \theta^{2}$ of the real line $\left(R^{1}, d t^{2}\right)$ and the unit circle $\left(S^{1}, d \theta^{2}\right)$. Here the warping function $m: R \rightarrow(0, \infty)$ is a smooth even function. If the Gaussian curvature is positive on the equator and decreasing on the upper half meridian $t^{-1}(0, \infty) \cap$ $\theta^{-1}(0)$, then the function $\varphi(\nu)$ is decreasing on $(\inf m, m(0))$.

Proof. Let $\widetilde{M}:=\left(R^{1} \times R^{1}, d \tilde{t}^{2}+m(\tilde{t})^{2} d \tilde{\theta}^{2}\right)$ denote the universal covering space of $M$. Choose any point $\tilde{p}$ on $\tilde{t}^{-1}(0)$. For each $\nu \in(\inf m, m(0))$, let $\alpha_{\nu}:[0, \infty) \rightarrow \widetilde{M}$ denote the geodesic emanating from the point $\tilde{p}=\alpha_{\nu}(0)$ with Clairaut constant $\nu$ and with $\left(\tilde{t} \circ \alpha_{\nu}\right)^{\prime}(0)<0$. From the Clairaut relation, we get $\angle\left((\partial / \partial \tilde{\theta})_{\tilde{p}}, \alpha_{\nu}^{\prime}(0)\right)=\cos ^{-1} \nu / m(0)$. Choose any $\nu_{1}<\nu_{2}$ with $\nu_{1}, \nu_{2} \in(\inf m, m(0))$. Since

$$
\cos ^{-1} \frac{\nu_{2}}{m(0)}<\cos ^{-1} \frac{\nu_{1}}{m(0)}
$$

it follows from Lemma 4.5 that $\alpha_{\nu_{1}}$ does not cross the domain bounded by the subarc of $\alpha_{\nu_{2}}$ and $\tilde{t}^{-1}(0) \cap \tilde{\theta}^{-1}\left[\tilde{\theta}(\tilde{p}), \tilde{\theta}(\tilde{p})+\varphi\left(\nu_{2}\right)\right]$. This implies that $\varphi\left(\nu_{1}\right) \geq \varphi\left(\nu_{2}\right)$. Therefore, $\varphi(\nu)$ is decreasing on $(\inf m, m(0))$.

\section{The cut locus of a point on $\widetilde{M}$}

Choose any point $q$ on $\widetilde{M}$ with $-t_{0}<\tilde{t}(q)<0$, where $t_{0}:=\sup \left\{t>0 \mid m^{\prime}(t)<0\right\}$. Without loss of generality, we may assume that $\tilde{\theta}(q)=0$. We consider two geodesics $\alpha_{\nu}$ and $\beta_{\nu}$ emanating from the point $q=\alpha_{\nu}(0)=\beta_{\nu}(0)$ with Clairaut constant $\nu>0$. Here we assume that

$$
\angle\left((\partial / \partial \tilde{t})_{q}, \alpha_{\nu}^{\prime}(0)\right)>\angle\left((\partial / \partial \tilde{t})_{q}, \beta_{\nu}^{\prime}(0)\right)
$$

Lemma 5.1 The two geodesics $\alpha_{\nu}$ and $\beta_{\nu}$ intersect again at the point $(\tilde{t}, \tilde{\theta})^{-1}(u, \varphi(\nu))$ if $\nu \in(\inf m, m(0))$, where $u:=-\tilde{t}(q)$. 
Proof. Suppose that $\nu \in(\inf m, m(0))$. Since $\alpha_{\nu}$ is tangent to the parallel $\operatorname{arc} \tilde{t}=-\xi(\nu)$, it follows from (3.5) that

$$
\tilde{\theta}\left(\alpha_{\nu}\left(s_{1}\right)\right)-\tilde{\theta}\left(\alpha_{\nu}(0)\right)=\int_{-\xi(\nu)}^{-u} \frac{\nu}{m \sqrt{m^{2}-\nu^{2}}} d t
$$

where $s_{1}:=\min \left\{s>0 \mid \tilde{t}\left(\alpha_{\nu}(s)\right)=-\xi(\nu)\right\}$, and

$$
\tilde{\theta}\left(\alpha_{\nu}\left(s_{2}\right)\right)-\tilde{\theta}\left(\alpha_{\nu}\left(s_{1}\right)\right)=\int_{-\xi(\nu)}^{u} \frac{\nu}{m \sqrt{m^{2}-\nu^{2}}} d t
$$

where $s_{2}:=\min \left\{s>0 \mid \tilde{t}\left(\alpha_{\nu}(s)\right)=u\right\}$. Hence, we obtain,

$$
\tilde{\theta}\left(\alpha_{\nu}\left(s_{2}\right)\right)-\tilde{\theta}\left(\alpha_{\nu}(0)\right)=\int_{-\xi(\nu)}^{u} \frac{\nu}{m \sqrt{m^{2}-\nu^{2}}} d t+\int_{-\xi(\nu)}^{-u} \frac{\nu}{m \sqrt{m^{2}-\nu^{2}}} d t .
$$

Since $m$ is an even function,

$$
\int_{-\xi(\nu)}^{u} \frac{\nu}{m \sqrt{m^{2}-\nu^{2}}} d t=\int_{-\xi(\nu)}^{0} \frac{\nu}{m \sqrt{m^{2}-\nu^{2}}} d t+\int_{-u}^{0} \frac{\nu}{m \sqrt{m^{2}-\nu^{2}}} d t
$$

holds. Therefore, by (5.1),

$$
\tilde{\theta}\left(\alpha_{\nu}\left(s_{2}\right)\right)-\tilde{\theta}\left(\alpha_{\nu}(0)\right)=2 \int_{-\xi(\nu)}^{0} \frac{\nu}{\sqrt{m^{2}-\nu^{2}}} d t=\varphi(\nu) .
$$

This implies that $\alpha_{\nu}$ passes through the point $(\tilde{t}, \tilde{\theta})^{-1}(u, \varphi(\nu))$. On the other hand, after $\beta_{\nu}$ is tangent to $\tilde{t}=\xi(\nu)$ at $\beta_{\nu}\left(s_{1}^{+}\right)$, where $s_{1}^{+}:=\min \left\{s>0 \mid \tilde{t}\left(\beta_{\nu}(s)\right)=\xi(\nu)\right\}$, the geodesic intersects $\tilde{t}=u$ again at $\beta_{\nu}\left(s_{2}^{+}\right)$, where $s_{2}^{+}:=\min \left\{s>s_{1}^{+} \mid \tilde{t}\left(\beta_{\nu}(s)\right)=u\right\}$. By the similar computation as above, we get

$$
\tilde{\theta}\left(\beta_{\nu}\left(s_{2}^{+}\right)\right)-\tilde{\theta}\left(\beta_{\nu}(0)\right)=\varphi(\nu) .
$$

This implies that $\alpha_{\nu}$ and $\beta_{\nu}$ pass through the common point $(\tilde{t}, \tilde{\theta})^{-1}(u, \varphi(\nu))$.

Lemma 5.2 The two geodesic segments $\left.\alpha_{\nu}\right|_{\left[0, s_{2}\right]}$ and $\left.\beta_{\nu}\right|_{\left[0, s_{2}^{+}\right]}$have the same length and its length equals $l(\nu)$, which is defined in Lemma 3.2. In particular, $s_{2}=s_{2}^{+}$. Here, $s_{2}$ and $s_{2}^{+}$denote the numbers defined in the proof of Lemma 5.1.

Proof. From (3.6), we have

$$
L\left(\left.\alpha_{\nu}\right|_{\left[0, s_{1}\right]}\right)=\int_{-\xi(\nu)}^{-u} \frac{m}{\sqrt{m^{2}-\nu^{2}}} d t
$$

and

$$
L\left(\left.\alpha_{\nu}\right|_{\left[s_{1}, s_{2}\right]}\right)=\int_{-\xi(\nu)}^{u} \frac{m}{\sqrt{m^{2}-\nu^{2}}} d t=\int_{-\xi(\nu)}^{0} \frac{m}{\sqrt{m^{2}-\nu^{2}}} d t+\int_{0}^{u} \frac{m}{\sqrt{m^{2}-\nu^{2}}} d t
$$


where $s_{1}$ denotes the number defined in the proof of Lemma 5.1. Since $m$ is even

$$
L\left(\left.\alpha_{\nu}\right|_{\left[s_{1}, s_{2}\right]}\right)=\int_{-\xi(\nu)}^{0} \frac{m}{\sqrt{m^{2}-\nu^{2}}} d t+\int_{-u}^{0} \frac{m}{\sqrt{m^{2}-\nu^{2}}} d t .
$$

Therefore, we get, by (3.8), (5.2) and (5.3),

$$
L\left(\left.\alpha_{\nu}\right|_{\left[0, s_{2}\right]}\right)=2 \int_{-\xi(\nu)}^{0} \frac{m}{\sqrt{m^{2}-\nu^{2}}} d t=l(\nu) .
$$

Analogously we have,

$$
L\left(\left.\beta_{\nu}\right|_{\left[0, s_{2}^{+}\right]}\right)=l(\nu)
$$

Lemma 5.3 Let $q$ be a point on $\widetilde{M}$ with $|\tilde{t}(q)| \in\left(0, t_{0}\right)$. Then, for any $\nu \in(\inf m, m(u)]$, where $u=-\tilde{t}(q),\left.\alpha_{\nu}\right|_{\left[0, s_{2}(\nu)\right]}$ and $\left.\beta_{\nu}\right|_{\left[0, s_{2}(\nu)\right]}$ are minimal geodesic segments joining $q$ to the point $(\tilde{t}, \tilde{\theta})^{-1}(u, \tilde{\theta}(q)+\varphi(\nu))$, and in particular, $\{(\tilde{t}, \tilde{\theta}) \mid \tilde{t}=u, \tilde{\theta} \geq \varphi(m(u))+\tilde{\theta}(q)\}$ is a subset of the cut locus of the point $q$. Here, $s_{2}(\nu):=\min \left\{s>0 \mid \tilde{t}\left(\alpha_{\nu}(s)\right)=u\right\}$ for each $\nu \in(\inf m, m(0))$.

Proof. Without loss of generality, we may assume that $\tilde{\theta}(q)=0$. We will prove that $\left.\alpha_{\nu}\right|_{\left[0, s_{2}(\nu)\right]}$ is a minimal geodesic segment joining $q$ to the point $\alpha_{\nu}\left(s_{2}(\nu)\right)=(\tilde{t}, \tilde{\theta})^{-1}(u, \varphi(\nu))$. Suppose that $\left.\alpha_{\nu_{0}}\right|_{\left[0, s_{2}\left(\nu_{0}\right)\right]}$ is not minimal for some $\nu_{0} \in(\inf m, m(u)]$. Here we assume that $\nu_{0}$ is the minimum solution $\nu=\nu_{0}$ of $\varphi(\nu)=\varphi\left(\nu_{0}\right)$.

Let $\alpha:[0, d(q, x)] \rightarrow M$ be a minimal geodesic segment joining $q$ to $x:=\alpha_{\nu_{0}}\left(s_{2}\left(\nu_{0}\right)\right)=$ $(\tilde{t}, \tilde{\theta})^{-1}\left(u, \varphi\left(\nu_{0}\right)\right)$. Hence, $\varphi\left(\nu_{1}\right)=\varphi\left(\nu_{0}\right)=\tilde{\theta}(x)$ and $\alpha$ equals $\left.\alpha_{\nu_{1}}\right|_{\left[0, s_{2}\left(\nu_{1}\right)\right]}$ or $\left.\beta_{\nu_{1}}\right|_{\left[0, s_{2}\left(\nu_{1}\right)\right]}$, where $\nu_{1} \in(\inf m, m(0))$ denotes the Clairaut constant of $\alpha$. By Proposition 4.6, $\varphi(\nu)=$ $\varphi\left(\nu_{0}\right)$ for any $\nu \in\left[\nu_{0}, \nu_{1}\right]$. Hence, by Lemmas 3.2 and 5.2 we get,

$$
s_{2}\left(\nu_{1}\right)=L(\alpha)=L\left(\left.\alpha_{\nu_{1}}\right|_{\left[0, s_{2}\left(\nu_{1}\right)\right]}\right)=L\left(\left.\alpha_{\nu_{0}}\right|_{\left[0, s_{2}\left(\nu_{0}\right)\right]}\right)=s_{2}\left(\nu_{0}\right) .
$$

This implies that $\left.\alpha_{\nu_{0}}\right|_{\left[0, s_{2}\left(\nu_{0}\right)\right]}$ is minimal, which is a contradiction, since we assumed that $\left.\alpha_{\nu_{0}}\right|_{\left[0, s_{2}\left(\nu_{0}\right)\right]}$ is not minimal. Therefore, by Lemma 5.2, for any $\nu \in(\inf m, m(u)]$, the geodesic segments $\left.\alpha_{\nu}\right|_{\left[0, s_{2}(\nu)\right]}$ and $\left.\beta_{\nu}\right|_{\left[0, s_{2}(\nu)\right]}$ are minimal geodesic segments joining $q$ to the point $(\tilde{t}, \tilde{\theta})^{-1}(u, \varphi(\nu))=\alpha_{\nu}\left(s_{2}(\nu)\right)$. In particular, the point $\alpha_{\nu}\left(s_{2}(\nu)\right)=\beta_{\nu}\left(s_{2}(\nu)\right)$ is a cut point of $q$.

Proposition 5.4 The cut locus of the point $q$ in Lemma 5.3 equals the set

$$
\{(\tilde{t}, \tilde{\theta})|\tilde{t}=u, \tilde{\theta} \geq| \varphi(m(u)) \mid\} .
$$

Here the coordinates $(\tilde{t}, \tilde{\theta})$ are chosen so as to satisfy $\tilde{\theta}(q)=0$.

Proof. By Lemma 5.3, geodesic segments $\left.\alpha_{\nu}\right|_{\left[0, s_{2}(\nu)\right]}$ and $\left.\beta_{\nu}\right|_{\left[0, s_{2}(\nu)\right]}$ are minimal geodesic segments for any $\nu \in(\inf m, m(u)]$. Hence their limit geodesics $\alpha^{-}:=\alpha_{\inf m}$ and $\beta^{+}:=$ $\beta_{\inf m}$ are rays, that is, any their subarcs are minimal. 
Since $\widetilde{M}$ has a reflective symmetry with respect to $\tilde{\theta}=0$, it is trivial from Lemma 5.3 that the set $\{(\tilde{t}, \tilde{\theta})|\tilde{t}=u, \tilde{\theta} \geq| \varphi(m(u)) \mid\}$ is a subset of the cut locus of $q$. Suppose that there exists a cut point $y \notin\{(\tilde{t}, \tilde{\theta})|\tilde{t}=u, \tilde{\theta} \geq| \varphi(m(u)) \mid\}$. Without loss of generality, we may assume that $\tilde{\theta}(y)>0=\tilde{\theta}(q)$ and $\tilde{t}(q)=-u<0$. From Lemma 4.5, $\tilde{t}(y)>0$ and $y$ is not a point in the unbounded domain cut off by two rays $\alpha^{-}$and $\beta^{+}$, and hence the point lies in the domain $D^{+}$cut off by $\beta^{+}$and the submeridian $\tilde{t}>-u, \tilde{\theta}=\tilde{\theta}(q)=0$. Since the cut locus of $C_{q}$ has a tree structure, there exists an end point $x$ of the cut locus in the $D^{+}$. Hence, $x$ is conjugate to $q$ for any minimal geodesic segment $\gamma$ joining $q$ to $x$. Since such a minimal geodesic $\gamma$ runs in the domain $D^{+}$, the Clairaut constant of the segment is positive and less than inf $m$. From the Clairaut relation (3.2), any geodesic cannot be tangent to any parallel arc $\tilde{t}=c$, if the Clairaut constant is positive and less than inf $m$. From Corollary 7.2.1 in [SST], $\gamma$ has no conjugate point of $q$, which is a contradiction.

Lemma 5.5 Let $q$ be a point on $\widetilde{M}$ with $|\tilde{t}(q)| \geq t_{0}$. Then the cut locus of $q$ is empty.

Proof. Suppose that the cut locus of a point $q$ with $|\tilde{t}(q)| \geq t_{0}$ is nonempty. Since $\widetilde{M}$ has a reflective symmetry with respect to $\tilde{t}=0$, we may assume that $\tilde{t}(q) \leq-t_{0}$. Hence by Lemma 4.5, there exists an end point $x$ of the cut locus $C_{q}$ in $\tilde{t}^{-1}(0, \infty)$. Let $\gamma:[0, d(q, x)] \rightarrow \widetilde{M}$ denote a minimal geodesic segment joining $q$ to $x$. Then $x$ is conjugate to $q$ along $\gamma$, since $x$ is an end point of $C_{q}$. Since $\tilde{\theta}(x)>0=\tilde{\theta}(q)$, the Clairaut constant $\nu$ of $\gamma$ is positive, by (3.1). Moreover, from the Clairaut relation (3.2), the Clairaut constant $\nu$ is less than $\inf m=m\left(t_{0}\right)$, since $\gamma$ intersects $\tilde{t}=-t_{0}$. Therefore, $\gamma$ cannot be tangent to any parallel arc $\tilde{t}=c$. From Corollary 7.2.1 in [SST], $\gamma$ has no conjugate point of $q$, which is a contradiction.

Now our Main theorem is clear from Proposition 5.4 and Lemma 5.5.

\section{Acknowledgments}

I would like to express my gratitude to Professor Minoru TANAKA who kindly gave me guidance for the lectures and numerous comments.

\section{References}

[Bh] Richard L. Bishop, Decomposition of cut loci, Proc. Amer. Math. Soc. 65 (1) (1977), 133-136.

[E] D. Elerath, An improved Toponogov comparison theorem for non-negatively curved manifolds, J. Differential Geom. 15 (1980), 187-216.

[IK] J. Itoh, K. Kiyohara, The cut locui and the conjugate loci on ellipsoids, Manuscripta Math. 114 (2004), 247-264.

[IT] J. Itoh, M. Tanaka, The Lipschitz continuity of the distance function to the cut locus, Trans. of AMS, 353 (1) (2000), 21-40. 
[ShT] K. Shiohama, M. Tanaka, Cut loci and distance spheres on Alexandrov surfaces, Séminaries \& Congrès, Collection SMF No.1, Actes de la table ronde de Géométrie différentielle en l'honneur Marcel Berger (1996), 531-560.

[SST] K. Shiohama, T. Shioya, and M. Tanaka, The Geometry of Total Curvature on Complete Open Surfaces, Cambridge tracts in mathematics 159, Cambridge University Press, Cambridge, 2003.

[ST] R. Sinclair, M.Tanaka, The cut locus of a two-sphere of revolution and Toponogov 's comparison theorem, Tohoku Math. J. 59 (2007) 379-399.

[Ta] K. Tamura, On the cut locus of a complete Riemannian manifold homeomorphic to a cylinder, 2003, Master Thesis, Tokai University.

[Ts] Y. Tsuji, On a cut locus of a complete Riemannian manifold homeomorphic to a cylinder, Proceedings of the school of Science, Tokai University, 32 (1997) $23-34$.

[WZ] R. L. Wheeden, A. Zygmund, Measure and Integral, Marcel Dekker, New York, Basel, 1977.

\author{
Pakkinee CHITSAKUL \\ Department of Mathematics \\ King Mongkut's Institute of Technology Ladkrabang \\ Ladkrabang, Bangkok \\ $10-520$ Thailand \\ kcpakkin@kmitl.ac.th
}

